\title{
Baseline Observation Carried Forward Imputation Technique
}

National Cancer Institute

\section{Source}

National Cancer Institute. Baseline Observation Carried Forward Imputation Technique. NCI Thesaurus. Code C81201.

The substitution of a missing data point with the value from the subject's baseline observation that is not missing. 